\title{
e-COVID Xingu: Mídias Sociais e Informação no Combate à Covid-19 em Altamira, Pará
}

\author{
e-COVID Xingu: Social Media and Information against Covid-19 in Altamira, Pará
}

\author{
Lucas Mendes Carvalho' (i), Felipe Azevedo Alberto Nascimento' (iD), Renan Rocha Granato' (iD)

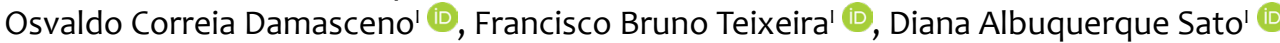

\section{RESUMO}

Introdução: A necessidade de levar informação visando combater a Covid-19 é ainda mais urgente nas regiões em desenvolvimento, uma vez que nesses locais há carência de recursos, supervisão governamental limitada, consideráveis índices de pobreza e dificuldade de acesso à informação. Com base na realidade da Transamazônica e do Xingu e na urgente necessidade da diminuição do número de casos da Covid-19 na região, a qual depende da adesão da população às medidas preventivas, surgiu o projeto de extensão "e-COVID Xingu: Mídias Sociais e Informação no Combate à COVID-19 em Altamira, Pará". Este trabalho tem como objetivo descrever as experiências dos integrantes desse projeto durante a pandemia na região.

Relato de Experiência: O projeto adotou como público-alvo a população do Xingu, em especial as comunidades indígenas e rurais. Publicações nas redes sociais levaram informações sobre medidas de prevenção, grupos de risco e isolamento social. Para alcançar as populações mais vulneráveis e que não possuem acesso à internet, uma parceria com a rádio local levou material informativo para a zona rural e comunidades indígenas afastadas. O projeto também lançou uma cartilha, em português e kayapó, com orientações de prevenção da Covid-19 para os indígenas do Médio Xingu.

Discussão: Os informativos conseguiram bom alcance pelas redes sociais. Ademais, os meios de comunicação em massa, como o rádio, ainda se mostram eficazes na disseminação de informações. Com multiplataformas pode-se fazer educação em saúde inclusiva a diversos grupos sociais, seja pela internet, pelo rádio ou por materiais físicos.

Conclusão: Utilizando múltiplas ferramentas de comunicação e respeitando o distanciamento social, a universidade, por meio de ação extensionista, pôde contribuir no combate à Covid-19, ao levar informações e conhecimento ao público, e atentar à necessidade de também incluir e informar populações histórica, social e economicamente vulneráveis, como indígenas e comunidade rurais do Xingu.

Palavras-chave: Infecções por Coronavírus; Educação em Saúde; Saúde das Populações Indígenas; Zona Rural; Meios de Comunicação em Massa.

\begin{abstract}
Introduction: The need to provide information in the fight against COVID-19 is more urgent in developing regions, which suffer from insufficient resources, limited government supervision, high poverty rates and difficulty in accessing information. Considering the reality experienced by those living by the Transamazonian Highway and the Xingu River and the urgent need to reduce COVID-19 cases in the region, which depends on the population taking prevention measures, this community outreach project called "e-COVID Xingu: Social media and information against COVID-19 in Altamira, Pará" took shape. This article aims to describe the experiences of the project members in the initial months of the outreach project during the pandemic in the region.

Experience Report: The project's audience target was the population of the Xingu region, especially indigenous communities and those living in rural areas. While posts on social media spread information about prevention, risk groups and social isolation, in order to reach the most vulnerable people, who lack an internet connection, a partnership with a local TV and radio broadcaster provided important information to these remote indigenous villages and rural communities. Moreover, an informative booklet with guidance on how to prevent infection by COVID-19 was released in both Portuguese and the Kayapo language to raise awareness and understanding among Xingu indigenous communities.
\end{abstract}

Discussion: The posts on social media achieved a good reach in social media. Furthermore, mass media, like radio, remain efficient information disseminators. The use of multiple tools is one possible way to educate diverse social groups, including the use of the internet, the radio or printed materials.

Conclusion: Using different communication platforms and respecting social distancing, the university, through this community outreach project, was able to contribute to the fight against COVID-19, spreading accurate and safe information and considering the need to include historically, socially and economically vulnerable population groups such as the indigenous and rural Xingu communities.

Keywords: Coronavirus Infections; Health Education; Health of Indigenous People; Rural Areas; Mass Media.

'Universidade Federal do Pará, Altamira, Pará, Brasil.

Correspondência

Diana Albuquerque Sato.

Universidade Federal do Pará, Faculdade de Medicina - Câmpus Universitário de Altamira, Avenida Coronel José Porfírio, número 2515, Centro, Altamira, PA, Brasil. CEP: 68371-040.

E-mail: disato@gmail.com

Recebido em 11/08/20; Aceito em 31/08/20 


\section{INTRODUÇÃO}

No Brasil, o primeiro caso confirmado do novo coronavírus ocorreu em São Paulo, no dia 26 de fevereiro de 2020' Na Amazônia brasileira, a doença causada pelo novo coronavírus (Covid-19) se alastrou rapidamente, indo do primeiro caso em Manaus, no Amazonas, no dia 13 de março, a mais de 400 mil casos confirmados na região, em julho de $2020^{2}$. Segundo a Secretaria de Estado de Saúde Pública do Pará, a região da Transamazônica (Altamira, Anapu, Brasil Novo, Medicilândia, Pacajá, Porto de Moz, Senador José Porfírio, Uruará e Vitória do Xingu) (Figura 1) foi de 300 casos confirmados em maio a mais de seis mil casos em junho de $2020^{3}$.

A região do Médio Xingu, mais especialmente Altamira, recebeu, entre 2010 e 2016, um enorme quantitativo de pessoas para trabalhar na construção da Usina Hidrelétrica (UHE) de Belo Monte, no rio Xingu. Entre os principais debates acerca dos impactos negativos vinculados à obra, estão o inchaço populacional e as consequentes problemáticas nas áreas da saúde, educação, criminalidade e construção de moradias em áreas de risco que colocam a cidade e a população em vulnerabilidade social ${ }^{4}$. Além disso, povos indígenas, ribeirinhos e moradores da zona rural de Altamira sofreram especial impacto com o advento da UHE Belo Monte. Essas mudanças ambientais e sociais consistem em redução dos níveis da água do rio Xingu no trecho abaixo da barragem principal, problemáticas para a navegação e os efeitos sobre a floresta aluvial em toda a área afetada pelo rebaixamento do lençol freático, extinção de espécies locais, escassez da pesca, aumento dos conflitos fundiários e do desmatamento, migração de não índios, ocupação desordenada do território, proliferação de epidemias e diminuição da qualidade da água 5 .

Outrossim, Altamira, como cidade referência na região, apresenta estrutura de serviços de saúde subdimensionada para uma população de aproximadamente 400 mil habitantes quando se consideram os nove municípios da região. A cidade, conhecida como a capital da Transamazônica, que já enfrentava antes da pandemia uma sobrecarga dos serviços de saúde ${ }^{6}$, está sujeita a uma saturação ainda maior de seus serviços médico-hospitalares, uma vez que, com o aumento dos casos de Covid-19, há aumento expressivo da necessidade de internação hospitalar e demanda por profissionais de saúde.

A necessidade de atuação na comunidade para promover informações sobre o combate à pandemia da Covid-19 é ainda mais urgente em regiões em desenvolvimento, uma vez que o cumprimento de tais contramedidas é deficitário nesses locais por causa de recursos insuficientes, supervisão governamental limitada, pobreza geral e dificuldade de acesso à informação? $\mathrm{E}$, apesar de todo o conhecimento científico desenvolvido ao longo dos últimos meses sobre a Covid-19, as medidas preventivas são ainda a melhor estratégia para limitar a

Figura 1. Mapa dos municípios da região do Médio Xingu, cuja administração dos serviços de saúde é realizada pelo $10^{\circ}$ Centro Regional.
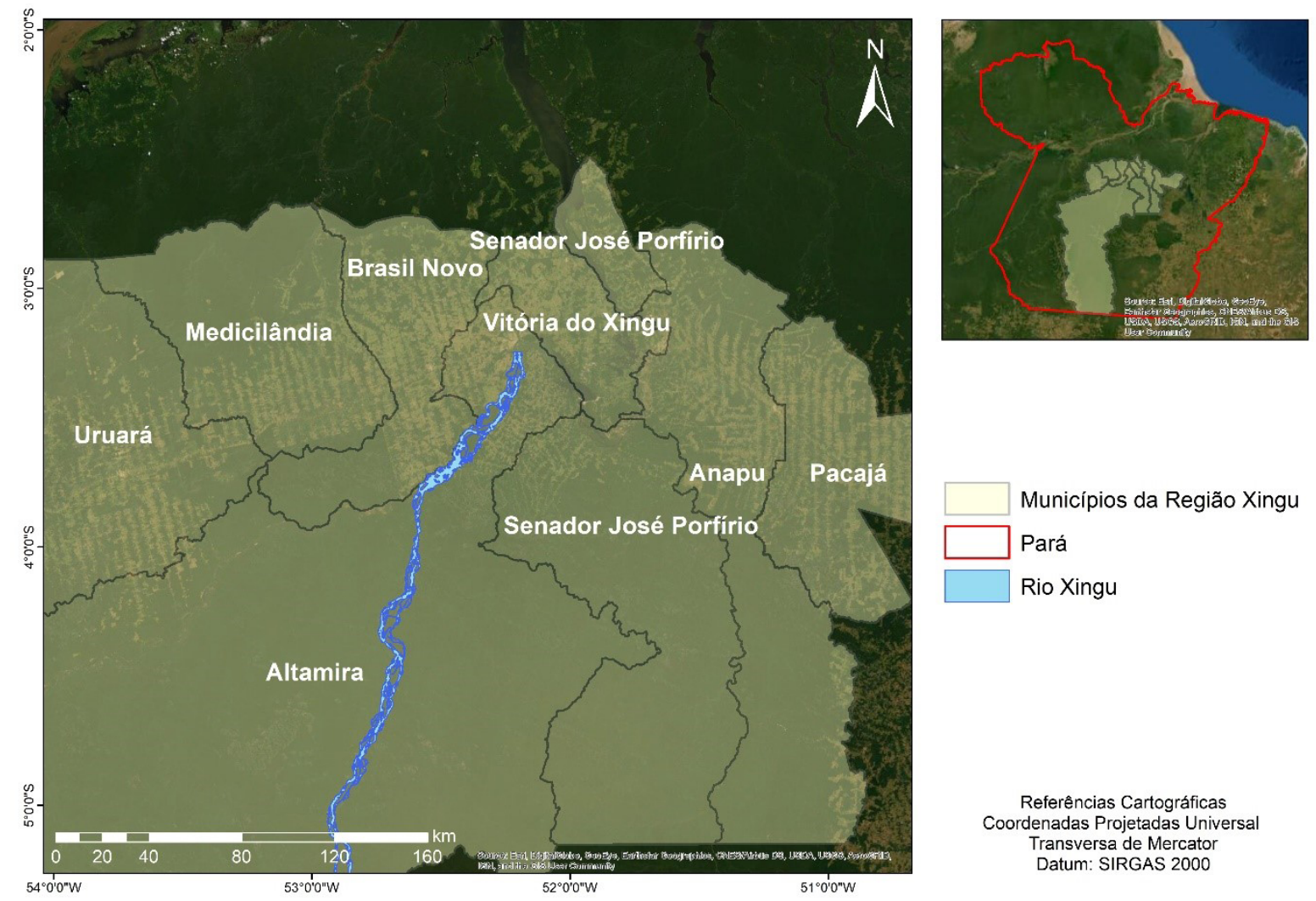

Fonte: João Kelvy Silva, Universidade Federal do Pará. 
progressão da pandemia e o colapso dos sistemas de saúde ${ }^{8,9}$.

Dessa forma, quando se considera a realidade local da Transamazônica e do Xingu, é essencial a diminuição da expansão do número de casos, a qual depende da adesão da população às medidas preventivas, tornando necessária a aplicação de ações de educação em saúde. Nesse contexto, surgiu o projeto de extensão "e-COVID Xingu: Mídias Sociais e Informação no Combate à COVID-19 em Altamira, Pará”. Alunos e professores da Faculdade de Medicina da Universidade Federal do Pará - câmpus Altamira - desenvolveram diversos materiais para levar informações de saúde à população, com especial atenção às mais vulneráveis. Postagens em redes sociais, lives com especialistas, programação na rádio com alcance nas mais longínquas localidades, distribuição de cartilha impressa e podcasts abordaram os principais temas sobre a Covid-19.

Este trabalho tem como objetivo descrever as experiências dos integrantes do projeto de extensão "e-COVID Xingu: Mídias Sociais e Informação no Combate à COVID-19 em Altamira, Pará" nos três primeiros meses de sua atuação durante a pandemia na região.

\section{RELATO DE EXPERIÊNCIA}

O projeto extensionista concentrou-se na educação em saúde acerca de medidas de prevenção à Covid-19 durante o período da pandemia do coronavírus no município de Altamira, no interior do Pará. O projeto teve como públicoalvo a população de Altamira e cidades da região do Médio rio Xingu e enfoque em populações vulneráveis como indígenas e moradores de comunidades rurais. Dadas as limitações impostas pela necessidade de distanciamento social, todo o projeto teve como palco as diversas redes sociais e o rádio, por meio dos quais o público local pôde ser atingido pelas campanhas. As campanhas on-line estiveram atreladas à divulgação de imagens, vídeos e transmissões ao vivo nas redes sociais Instagram, Facebook e WhatsApp. Além disso, as campanhas foram adaptadas para inserções durante programas de rádio.

Para a realização do projeto, foi reunida uma equipe com discentes e docentes da Faculdade de Medicina da Universidade Federal do Pará - câmpus Altamira, os quais estiveram envolvidos na produção de campanhas, cartilhas informativas, vídeos, textos para o rádio e transmissões ao vivo. No início do projeto, definiu-se a realização de reuniões online quinzenais para a discussão de estratégias de abordagem de temas, produção de campanhas, orientações sobre temas relativos à Covid-19, além do acompanhamento sobre o alcance das postagens e o público atingido. Dessa forma, as reuniões definiram os temas das campanhas de acordo com o avanço do vírus e as demandas espontâneas de grupos da comunidade. Ressalta-se que, para a aferição de impacto das diversas campanhas, foram utilizados os recursos de análise presentes em cada uma das plataformas sociais utilizadas.

Após a seleção de tema e análise dos dados de impacto das campanhas, ocorreu a produção de banners digitais (Figura 2) para veiculação simultânea via Instagram, Facebook e WhatsApp, e para a produção de textos para inserções diárias em programas de parceiros do projeto.

Para levar essas informações de maneira inclusiva a quem não tem acesso à internet, seja na cidade ou nas remotas áreas rurais do maior município brasileiro em extensão territorial, Altamira, a transmissão pela rádio se fez eficiente. Levou-se em conta também o grande sucateamento do sistema educacional na região da transamazônica, que desde o último levantamento do Instituto Brasileiro de Geografia e Estatística (IBGE) possuía um considerável quantitativo de não alfabetizados ${ }^{10}$. Como os materiais para as mídias sociais são escritos, a rádio é, desse modo, muito eficaz para que o conteúdo informativo alcance os mais variados públicos. A produção do texto é feita pelos

Figura 2. Banners digitais ( $A, B$ e $C$ ) sobre recomendações para comunidades indígenas produzidos para veiculação no Instagram, Facebook e WhatsApp.

A

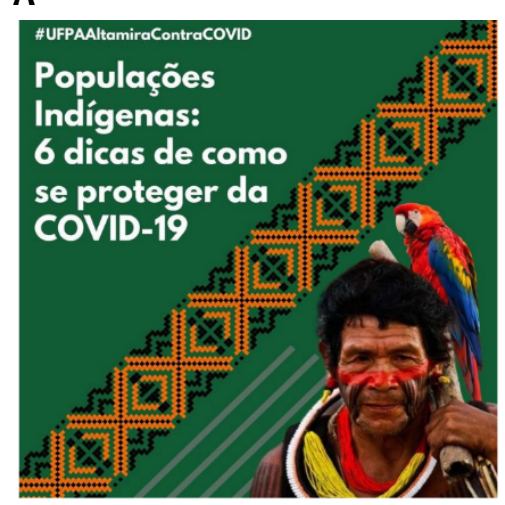

B

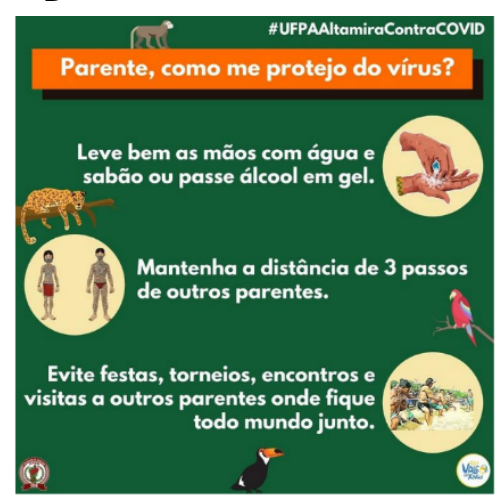

C

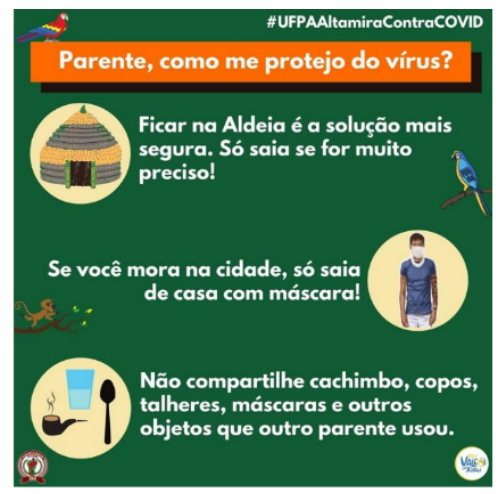

Fonte: Acervo do projeto de extensão. 
próprios participantes do projeto e enviada semanalmente para a rádio local. O material é lido pelo locutor durante os intervalos dos programas diariamente, desde a madrugada até a última programação da noite.

Com o avanço do coronavírus pelas aldeias da Amazônia, os participantes do projeto, com a colaboração de alunos indígenas da Faculdade de Medicina da Universidade Federal do Pará, desenvolveram uma cartilha informativa voltada àquelas que podem ser as mais vulneráveis da pandemia: as comunidades indígenas (Figura 3). A cartilha lançada em 29 de junho trouxe temas como a importância do isolamento social, grupos de riscos, como fazer a lavagem correta das mãos, medidas de prevenção, uso de máscaras, sinais e sintomas da Covid-19 e quando procurar ajuda médica (Figura 4). Com versões em português e kayapó, linguagem simples e acessível, e elementos que identificam culturalmente as comunidades

Figura 3. Capa da cartilha informativa com orientações preventivas sobre a Covid-19 para indígenas do Xingu. (A) Capa da versão em língua portuguesa. (B) Capa da versão em língua indígena kayapó.

A

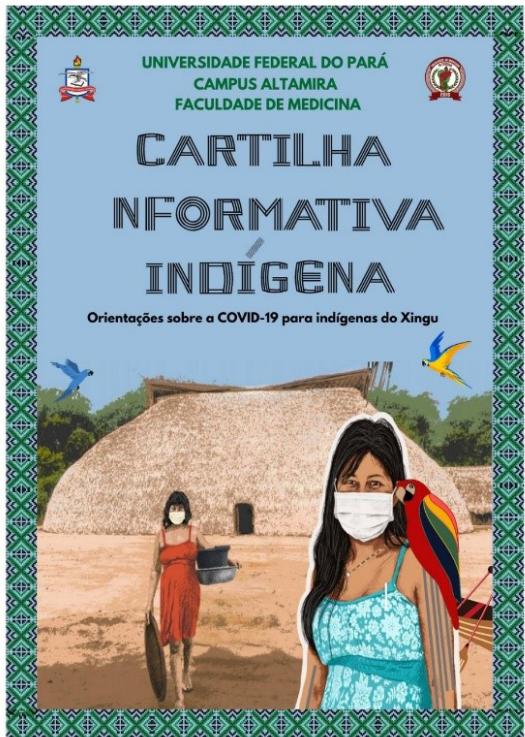

B

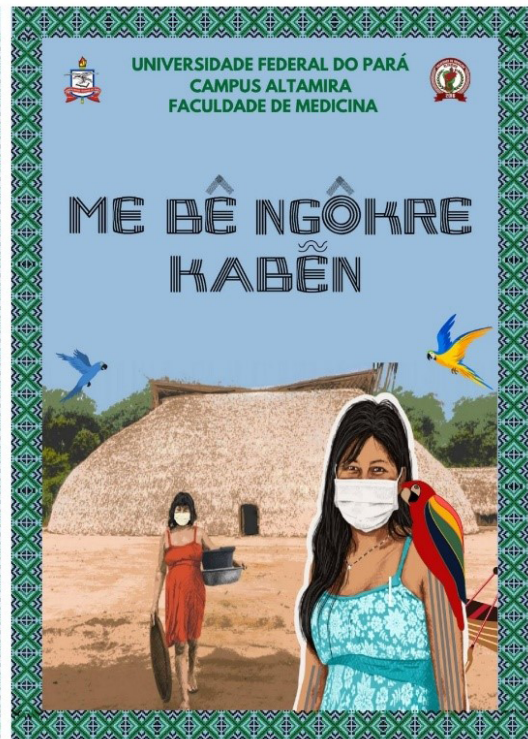

Fonte: Acervo do projeto de extensão.

Figura 4. Seção da cartilha informativa voltada para indígenas do Xingu que aborda medidas de prevenção contra a Covid-19. (A) Seção da versão em língua portuguesa. (B) Seção da versão na língua indígena kayapó.

A

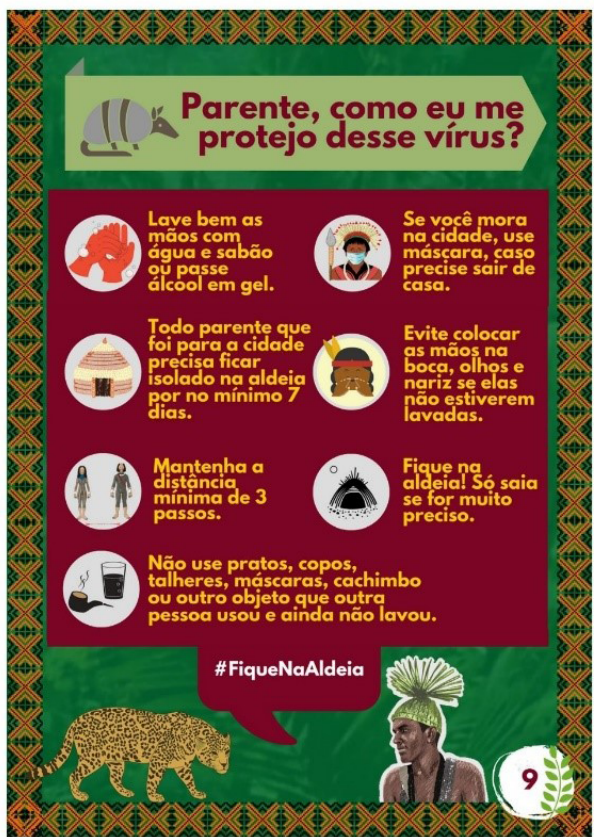

B

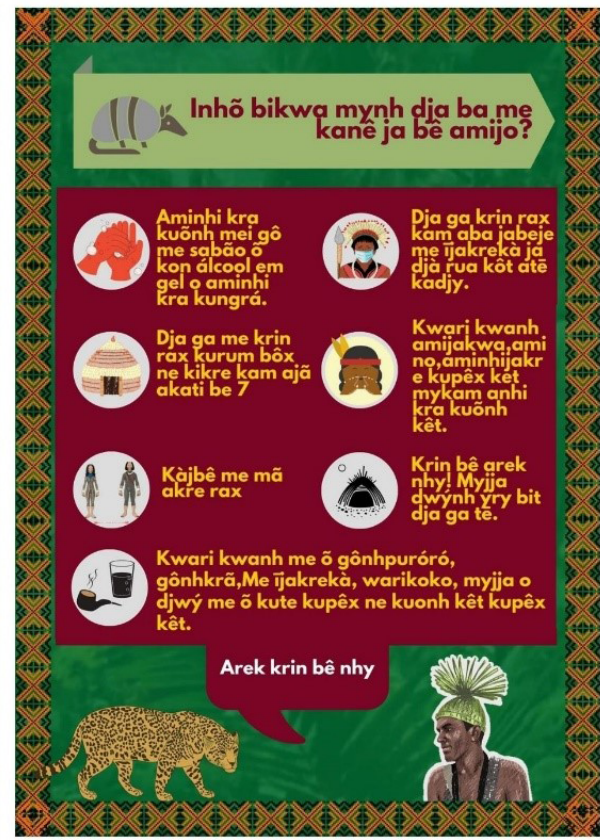

Fonte: Acervo do projeto de extensão. 
indígenas da região, a cartilha foi distribuída na versão impressa, PDF e em podcast para as aldeias do Xingu por meio de uma parceria com o Instituto Socioambiental (ISA).

Ainda havia a seleção de temas para a produção de vídeos, os quais eram produzidos por docentes ou discentes da Faculdade de Medicina de Altamira. Além disso, eram selecionados temas para a realização de transmissões ao vivo na plataforma YouTube com profissionais da saúde locais. Para o engajamento do público, sempre era realizada a divulgação das transmissões por meio das redes sociais com ao menos dois dias de antecedência, e, durante cada transmissão, o público era incentivado a participar das discussões por meio de comentários e envio de perguntas sobre o tema tratado.

\section{DISCUSSÃO}

A limitação imposta pela pandemia da Covid-19 à realização de atividades de educação em saúde presenciais levou à necessidade da adaptação destas ao ambiente virtual. Segundo Cuello-Garcia, Gaxiola-Pérez et al. ${ }^{11}$, o uso de redes sociais como foco de disseminação de conhecimento em saúde pode diminuir as lacunas da transmissão de conhecimentos em saúde do meio acadêmico para a sociedade civil e, assim, tornar-se uma ferramenta de fomentação de medidas e práticas benéficas à saúde pública como no caso da pandemia da Covid-19. Seguindo tal tendência, ao longo de três meses o projeto levou informações sobre prevenção e práticas contra a Covid-19 por meio das redes sociais para o município de Altamira (Pará) e cidades circunvizinhas da região do Médio e Baixo Xingu.

A definição da realização de reuniões quinzenais para o planejamento de ações e avaliação das atividades realizadas mostrou-se essencial para o bom andamento do projeto e alcance de objetivos propostos. Enquanto surgiam novas fases do avanço do vírus na região, as campanhas adaptaram-se à realidade local ao buscarem atender às demandas de grupos vulneráveis e alertarem a população sobre boas práticas e os riscos existentes. Além disso, as reuniões permitiram a discussão ativa de temas e do panorama da pandemia na região entre docentes e discentes da Faculdade de Medicina, o que fomentou a voz ativa de discentes na discussão sobre a saúde pública local.

Uma importante frente de disseminação de informações para a população em geral da região foi por meio da produção de banners informativos para as redes sociais Instagram, Facebook e WhatsApp. As postagens tiveram sua veiculação realizada com frequência nas redes sociais mencionadas e abordaram temas por meio de banners digitais com ilustrações, textos claros e indicação de contato para eventuais dúvidas. Os temas incluíram o estímulo à adesão de práticas como etiqueta respiratória, distanciamento social, uso e fabricação de máscaras de tecido, como agir em caso de suspeita de Covid-19; informações sobre sobre o lockdown na cidade de Altamira, diferenciação de coronavírus, Sars-CoV-2 e Covid-19, sintomas e sinais de alerta da Covid-19, pré-natal e amamentação durante a pandemia e recomendações para a população indígenas. Essas postagens com banners temáticos tiveram um alcance total, nas redes sociais Instagram e Facebook, de 15.735 pessoas, ou seja, considerando a realização de 20 campanhas em forma de banner, a média de alcance por campanha foi de 786 pessoas.

Além disso, com a pulverização de comunidades rurais pelo território do Xingu, o acesso à serviços básicos de saúde por essa população se torna difícil. Como agravante, podem-se adicionar o pequeno número de equipe de saúde da família por toda a Amazônia, o longo tempo de espera para atendimentos, muitas vezes realizados apenas nas cidades, e a ausência da oferta de atendimento domiciliar a essas regiões remotas ${ }^{12,13}$. Tais dificuldades geográficas e logísticas colocam os moradores da zona rural da Transamazônica em situação vulnerável. Outrossim, apesar do isolamento geográfico, na América Latina como um todo, e em especial no Brasil, ocorreu a interiorização da Covid-19. As comunidades rurais foram impactadas pela pandemia e sofrem consequências desproporcionais por conta da carência de recursos de saúde e infraestrutura ${ }^{14}$.

Levando tais pontos em consideração, como estratégia adjuvante para que a informação chegue àqueles que não possuem acesso à internet, seja na cidade ou nas áreas rurais do Xingu e, também, aos que não sabem ler ou possuam deficiência visual, a transmissão via rádio foi efetivada. Altamira é o maior município brasileiro em extensão territorial, sendo maior que países como Portugal e Grécia'5 ${ }^{15}$ Com tamanha dimensão, é notória a presença de comunidades rurais nas mais afastadas áreas do município. A informação chega facilmente a essas localidades por meio da rádio. Assim, em parceria com a Rede de Rádio e TV Vale do Xingu, semanalmente várias orientações sobre prevenção e cuidados na pandemia foram veiculadas pela transmissora de rádio (93.1 FM).

Com textos diariamente veiculados pela rádio, o projeto de extensão, por meio da divulgação de informação em saúde a áreas onde há ausência de serviços da atenção básica, conseguiu uma boa aderência do público das zonas rural e urbana. Como os programas de rádio permitem que o ouvinte ligue e interaja com o locutor ao vivo, houve muitos comentários sobre os informativos, demonstrando a eficácia da chegada do conteúdo às localidades remotas do Xingu, sem deixar de alcançar o público não usuário da internet na cidade, os deficientes visuais e também os não alfabetizados que, sem a rádio, não teriam acesso ao conteúdo das postagens escritas.

O enfoque nas populações vulneráveis fomentado pelas discussões entre os membros do projeto é de extrema 
importância quando se considera que o público pertencente às comunidades indígenas e rurais está sujeito à falta de serviços de saúde por causa das disparidades socioeconômicas e da ausência de informações. No início de maio, projeções referentes à população indígena na Amazônia legal já sinalizavam que o Distrito Sanitário Especial Indígena (DSEI) de Altamira estava entre as regiões com risco imediato de epidemia pelo novo coronavírus, já com casos registrados e risco de transmissão comunitária ${ }^{16}$. Além disso, para o combate à disseminação da Covid-19, desafios como a descrença nas equipes de saúde e autoridades, além boatos e informações falsas, buscaram ser superadosporesseprojetocomadisseminação, nascomunidades indígenas e rurais, de informações com embasamento científico e abordagens culturalmente adequadas ${ }^{17}$.

Com isso, a produção de uma cartilha informativa voltada para as comunidades indígenas do Médio Xingu mostrou-se relevante. Desde a implementação da UHE Belo Monte, os impactos socioeconômicos e ambientais deixaram grande parcela desses habitantes da Transamazônica em vulnerabilidade. Com a construção da barragem, territórios indígenas foram inundados, forçando o deslocamento em massa dessa população para outras áreas. O empreendimento impactou a qualidade da água, provocou a extinção de algumas espécies de peixes e alterou rotas de pesca. Com a inundação desses territórios, houve um deslocamento de parte dessa população para a cidade de Altamira, gerando agora um impacto identitário-cultural ${ }^{18}$. Além desses fatores, podem-se citar as pequenas moradias indígenas com múltiplos moradores, as dificuldades de acesso a medidas preventivas, como água limpa, sabão e álcool em gel, além da grande distância dessas comunidades dos serviços de saúde, o que contribui para o efeito devastador desproporcional da Covid-19 nos indígenas ${ }^{19}$.

De forma a incluir o máximo de público com tais informações, foram disponibilizadas versões em português e kayapó, língua falada por parte da comunidade indígena do $\mathrm{Xingu}^{20}$. Além disso, segundo a Teoria do Aprendizado Multimídia, a capacidade de aprendizado é maior em conteúdos de áudio do que por leitura ${ }^{21}$. Levando esse aspecto em consideração e visando a uma melhor aplicação dos conhecimentos preventivos, uma versão da cartilha em formato de podcast foi distribuída para o ISA, o qual possui membros e colaboradores presentes diariamente nas aldeias. Assim, esses profissionais puderam disponibilizar o conteúdo em áudio, a fim de dinamizar e facilitar o acesso à informação preventiva. O ISA também patrocinou a versão impressa distribuída para os indígenas.

Desse modo, com as mais diversas ferramentas foi possível aplicar educação em saúde a uma população historicamente prejudicada e que sofre na atualidade as mazelas causadas pelos grandes empreendimentos. Com temas que elucidam e explicam de forma acessível sobre grupos riscos, quando procurar ajuda médica, como lavar as mãos e a importância do isolamento social, foi possível se contrapor à onda de desinformação e notícias falsas que circulam no país, mesmo em época de pandemia, uma vez que sem a disseminação de informação verdadeira e científica os esforços para o enfrentamento da Covid-19 podem acabar mitigados ${ }^{22}$.

Outra linha de ação do projeto foram as transmissões ao vivo, as quais ocorreram por meio das plataformas do YouTube e Facebook e objetivaram atingir públicos diversos. Ao longo de três meses, foram realizadas oito transmissões ao vivo, sete via YouTube e uma via Facebook. Cada transmissão teve sua divulgação prévia por meio das redes sociais Instagram, Facebook e WhatsApp. Os temas das transmissões incluíram: "Panorama Atual e Análise Epidemiológica da COVID-19 em Altamira e no Xingu”; "COVID-19: Testes Diagnósticos x Diagnóstico Clínico"; “Perguntas \& Respostas - COVID-19 em Foco"; "Cuidados com a Saúde Mental dos Idosos na Pandemia da COVID-19"; "Gestação e a COVID-19"; "Crianças e a COVID-19: Impactos, Vida Escolar e Perspectivas"; "Autocuidado na Pandemia: Alimentação e Atividade Física"; e "Quarentena e Saúde Mental: Como a psicologia pode ajudar?". Para a apresentação dos temas, houve a participação de um membro fixo do projeto com os convidados que incluíram epidemiologistas, pediatras, dentistas, médicos ginecologistas, enfermeiros, educadores físicos, psicólogos, biomédicos e farmacêuticos. Cada transmissão contou com a discussão do tema, apresentação de novas informações e participação do público por meio do envio de dúvidas e comentários acerca da discussão. A análise de dados das transmissões mostrou um total de 4.360 visualizações, com uma média de 545,1 visualizações a cada transmissão.

Outro enfoque do projeto foi a produção de vídeos curtos e objetivos sobre temas importantes para a prevenção da Covid-19. O formato adotado incluiu vídeos de no máximo cinco minutos produzidos por docentes e discentes, objetivando o fácil compartilhamento via aplicativos de mensagem e rápida visualização para adesão às práticas propostas e ao combate à desinformação. Os vídeos foram veiculados no Instagram, Facebook e YouTube e trataram de temas seguindo os títulos "Isolamento Domiciliar: Como e Quando Fazer?”, “Alerta COVID-19”, “COVID-19: Cuidados e Medidas de Prevenção” e “Medidas de Prevenção \& Cuidado Individual". Os vídeos tiveram um total de 722 visualizações ou uma média de 180 visualizações a cada vídeo.

Ao longo dos três meses, o projeto alcançou ao menos 39.048 pessoas (Gráfico 1), considerando o impacto aferido nas redes sociais Instagram, Facebook e YouTube de todo 
Gráfico 1. O alcance do total dos conteúdos produzidos e publicados nas redes sociais ao longo dos três meses.

\section{Número de contas alcançadas}

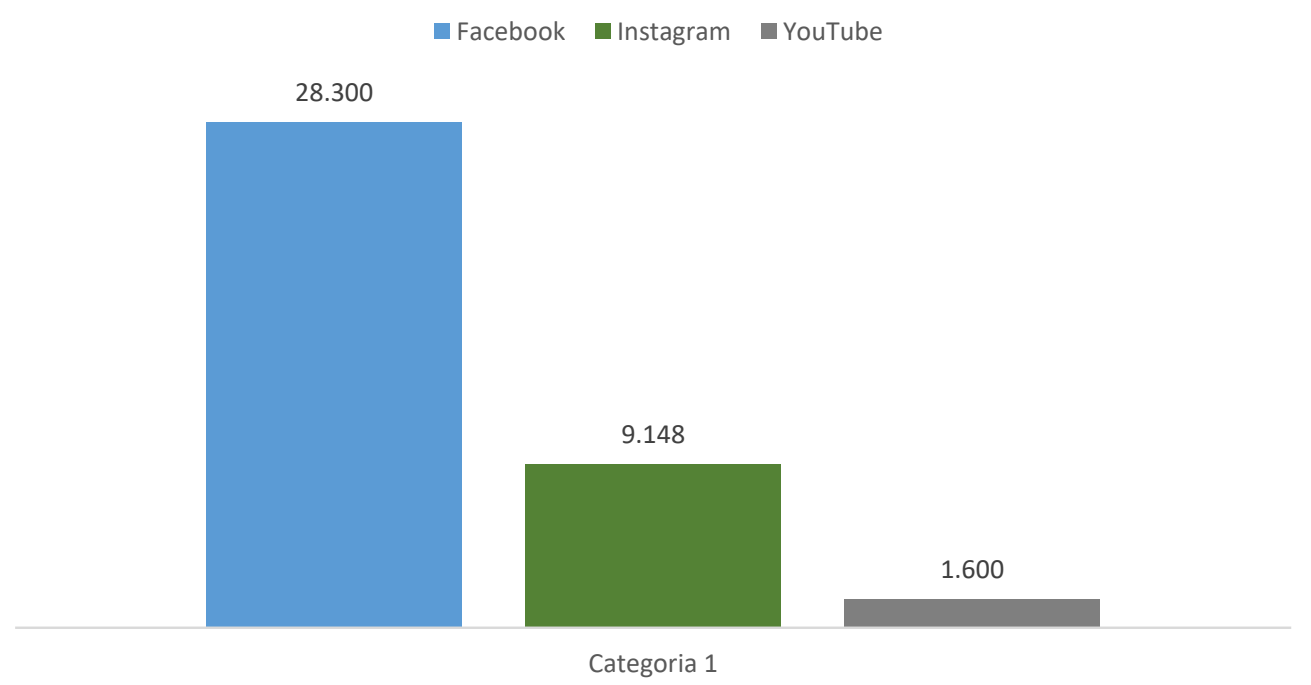

Fonte: Acervo do projeto de extensão

o conteúdo produzido, incluindo banners digitais, vídeos e transmissões ao vivo. Além disso, na rede social Instagram as campanhas tiveram maior repercussão em Altamira e Belém e predominância de audiência do público feminino.

\section{CONCLUSÃO}

A pandemia da Covid-19 evidenciou a necessidade de combate à desinformação, promoção de boas práticas, assistência à população local e enfrentamento dos desafios por comunidades em vulnerabilidade da região de Altamira e do Xingu. Dessa forma, a atuação do projeto de forma remota foi bem-sucedida ao atingir uma parcela da população e envolvêla na discussão por meio das estratégias implementadas. Ainda, provaram-se extremamente proveitosas as estratégias de atuação em comunidades sem acesso à internet, como por meio dos programas de rádio e entrega de cartilhas informativas impressas.

Por meio desses métodos, foi possível incluir coletivamente desde os usuários urbanos da internet até as comunidades indígenas vulneráveis do Médio Xingu, levando também material educativo de prevenção à zona rural da Transamazônica. O conteúdo beneficiou, em conjunto, a população não alfabetizada e os deficientes visuais, que, por meio dos programas na rádio, tiveram acesso às mesmas informações. Todo esse cerne de atuação possibilitou ainda uma aproximação entre a comunidade local e a comunidade acadêmica da universidade, o que gerou reconhecimento e valorização do trabalho desenvolvido pela universidade pública. Destarte, a atividade de extensão universitária promove a todos os atores sociais da região a possibilidade de desenvolver autonomamente práticas de prevenção contra a Covid-19 e, assim, auxiliar no enfrentamento do vírus.

\section{CONTRIBUIÇÃO DOS AUTORES}

Idealização do projeto que originou o relato de experiência e concepção e planejamento do estudo: Diana Albuquerque Sato.

Confecção de materiais e suporte técnico no desenvolvimento das ações: Lucas Mendes Carvalho e Felipe Azevedo Alberto Nascimento.

Revisão crítica e orientação na confecção de materiais e participação no repasse de informações ao público-alvo: Renan Rocha Granato, Osvaldo Correia Damasceno, Francisco Bruno Teixeira e Diana Albuquerque Sato.

Coleta e interpretação dos dados quantitativos: Lucas Mendes Carvalho, Felipe Azevedo Alberto Nascimento, Francisco Bruno Teixeira e Diana Albuquerque Sato.

Redação do manuscrito: Lucas Mendes Carvalho e Felipe Azevedo Alberto Nascimento.

Revisão crítica do manuscrito: Renan Rocha Granato, Osvaldo Correia Damasceno, Francisco Bruno Teixeira e Diana Albuquerque Sato.

Todos os autores aprovaram a versão final do manuscrito e assumem publicamente a responsabilidade pelo conteúdo do artigo.

\section{CONFLITO DE INTERESSES}

Os autores declaram não haver conflito de interesses neste relato. 


\section{REFERÊNCIAS}

1. Brasil. Brasil confirma primeiro caso da doença. Ministério da Saúde; 2020 [acesso em 6 ago 2020]. Disponível em: https://www.saude.gov. br/noticias/agencia-saude/46435-brasil-confirma-primeiro-caso-de-novocoronavirus.

2. Brasil. Painel Coronavírus [acesso em 7 ago 2020]. Disponível em: https:// covid.saude.gov.br/.

3. Pará. Secretaria Estadual de Saúde do Pará. COVID-19 [acesso em 8 ago 2020]. Disponível em: http://www.saude.pa.gov.br/coronavirus/.

4. Franco SV, Souza BE, Lima MA. Cheias e vulnerabilidade social: estudo sobre o rio Xingu em Altamira/PA. Ambiente e Sociedade. 2018;21:e01573.

5. Fearnside P. Belo Monte como ponta de lança 1: os impactos da primeira barragem. Amazônia Real. 2014 [acesso em 6 ago 2020]. Disponível em: http://philip.inpa.gov.br/publ_livres/2014/Belo_Monte-Ponta_de_ Lan\%C3\%A7a-1-A_primeira_barragem.pdf.

6. Instituto Brasileiro de Geografia e Estatística. Censo demográfico brasileiro. Rio de Janeiro: IBGE; 2010.

7. Rahimi F, Abadi ATB. Transparency and information sharing could help abate the COVID-19 pandemic. Infect Control Hosp Epidemiol. 2020;(22):1-2.

8. Cascella M, Rajnik M, Cuomo A, Dulebohn Sc, Di Napoli R. Features, evaluation and treatment coronavirus (COVID-19). Treasure Island, FL: StatPearls; 2020 [acesso em 6 ago 2020]. Disponível em: https://www. ncbi.nlm.nih.gov/books/NBK554776/

9. Organização Mundial Da Saúde. Coronavirus disease (COVID-19) advice for the public [acesso em 6 ago 2020]. Disponível em: https://www.who. int/emergencies/diseases/novel-coronavirus-2019/advice-for-public.

10. Instituto Brasileiro de Geografia e Estatística. Indicadores Sociais Municipais [acesso em 6 ago 2020]. Disponível em: https://cidades.ibge. gov.br/brasil/pa/altamira/pesquisa/23/25124.

11. Cuello-Garcia C, Gaxiola-Pérez G, Amelsvoort L. Social media can have an impact on how we manage and investigate the COVID-19 pandemic. J Clin Epidemiol. 2020 Jun 27 [Epub ahead of print]. [acesso em 6 ago 2020]. Disponível em: https://www.ncbi.nlm.nih.gov/pmc/articles/PMC7320665/

12. Garnelo L, Lima JL, Rocha ESC, Herkrath FJ. Acesso e cobertura da Atenção Primária à Saúde para populações rurais e urbanas na região norte do Brasil. Saúde Debate. 2018;42:81-99 [acesso em 5 ago 2020]. Disponível em: https://www.scielo.br/pdf/sdeb/v42nspe1/0103-1104-sdeb42-spe01-0081.pdf.
13. Floss M, Franco MC, Malvezzi C, Silva KV, Costa RB, Lima e Silva VX, et al. A pandemia de COVID-19 em territórios rurais e remotos: perspectiva de médicas e médicos de família e comunidade sobre a atenção primária à saúde. Cad Saude Publica 2020;36(7):e00108920.

14. Torrado S, Molina FR, Fowks J, Gortazar NG, Corona S. Comunidades rurais da América Latina enfrentam o avanço do coronavírus. El País. 2020 [acesso em 5 ago 2020]. Disponível em: https://brasil.elpais.com/ sociedade/2020-06-08/comunidades-rurais-da-america-latina-enfrentamo-avanco-do-coronavirus.html.

15. Instituto Brasileiro de Geografia e Estatística. Cidade de Altamira [acesso em 5 ago. 2020]. Disponível em: https://cidades.ibge.gov.br/brasil/pa/ altamira/panorama.

16. Codeço CT, Villela D, Coelho F, Bastos LS, Carvalho LM, Gomes MFC, et al. Risco de espalhamento da COVID-19 em populações indígenas: considerações preliminares sobre vulnerabilidade geográfica e socioeconômica. Rio de Janeiro: Fundação Osvaldo Cruz; 2020 [acesso em 10 ago 2020]. Disponível em: https://portal.fiocruz.br/sites/portal. fiocruz.br/files/documentos/relatorios_tecnicos_-_covid-19_proccemap-ensp-covid-19-report4_20200419-indigenas.pdf.

17. Meneses-Navarro S, Freyermuth-Enciso MG, Pelcastre-Villafuerte BE, Campos-Navarro R, Meléndez-Navarro DM, Gómez-Flores-Ramos L. The challenges facing indigenous communities in Latin America as they confront the COVID-19 pandemic. Int J Equity Health. 2020;19:63.

18. Araújo MMV, Pinto JK, Mendes FO. A Usina de Belo Monte e os impactos nas terras indígenas. Planeta Amazônia. 2014;(4):43-51.

19. Curtice K, Choo E. Indigenous populations: left behind in the COVID-19 response. Lancet. 2020 6-12 June;395(10239):1753.

20. Instituto Brasileiro de Geografia e Estatística. Os indígenas no Censo Demográfico 2010 [acesso 6 ago 2020]. Disponível em: https://indigenas. ibge.gov.br/images/indigenas/estudos/indigena_censo2010.pdf.

21. Guerch CA. Teoria da Carga Cognitiva e Teoria Cognitiva da Aprendizagem Multimídia: como utilizar ferramentas web na produção de materiais didáticos. Universidade Federal de Santa Maria; 2016 [acesso em 6 ago 2020]. Disponível em: https://educere.bruc.com.br/arquivo/ pdf2017/25739_13137.pdf.

22. Neto M, Gomes TO, Porto FR, Rafael RMR, Fonseca MHS, Nascimento J. Fake news no cenário da pandemia de Covid-19. Cogitare Enferm. 2020;25:e72627. 